\title{
Eleven Mutations in Six Genes Causing Abnormal Sperm Morphology: A Case Series
}

\section{Chongfu Zhong}

Shandong University of Traditional Chinese Medicine Affiliated Hospital https://orcid.org/0000-0003-2828-0959

Shengjing Liu

China Academy of Chinese Medical Sciences Xiyuan Hospital

Yong Wang

Affiliated Hospital of Jining Medical University

Zhuozhuo Shi

Shandong University of Traditional Chinese Medicine Affiliated Hospital

Fengrui Liu

Shandong University of Traditional Chinese Medicine

Zhaowang Gao ( $\sim$ liushengjing@88.com )

Shandong University of Traditional Chinese Medicine Affiliated Hospital

\section{Case Report}

Keywords: mutation, male infertility, sperm morphological abnormalities, next-generation sequencing

Posted Date: August 27th, 2021

DOl: https://doi.org/10.21203/rs.3.rs-783527/v1

License: (c) (1) This work is licensed under a Creative Commons Attribution 4.0 International License. Read Full License 


\section{Abstract}

Objective. Infertility has become a major health problem worldwide, approximately $10 \%-15 \%$ of male infertility cases can be attributed to genetic mutations. However, the genetic factors contributing to male infertility remain largely unknown. This study aimed to identify genetic mutations associated with morphological abnormalities in sperm.

Methods. In this study, we assessed the semen parameters of 60 patients with sperm morphological abnormalities by semen analysis, and subsequently performed targeted next-generation sequencing (NGS) to identify gene mutations.

Results. Of the 60 cases of male infertility, 8 individuals (13\%) were identified as carrying genetic mutations. In brief, patient 1 carried two heterozygous mutations of SUN5, including c.1066C>T and c.638delC; Patient 2 and Patient 3 had a 109.73 kb homozygous deletion on chromosome 12 in DPY19L2; patient 4 presented two heterozygous mutations (c.1670_1671delinsTT and c.255_258del) of DPY19L2; patient 5 carried a homozygous variant c.1159+1G>A of $C C D C 40$; patient 6 had a heterozygous mutation c.38_43del in the SEPT12 gene; patient 7 had two heterozygous mutations in DNAH1 (c.10060_10061insATCT and c.12278T>C), and patient 8 presented two heterozygous mutations of CFAP43 (c.3658C>T and c.823del).

Conclusions. We identified gene mutations associated with sperm morphological abnormalities in a series of male infertility cases. Eight of sixty individuals were identified as carrying gene mutations. Genetic mutations of SUN5, DPY19L2, CCDC40, SEPT12, DNAH1, and CFAP43 might be responsible for male infertility. Our study expanded the landscape of gene mutations related to sperm morphological abnormalities, which might provide new insights into the genetics underlying male infertility.

\section{Introduction}

According to the World Health Organization (WHO), infertility is defined as a failure to achieve a clinical pregnancy after 12 months or more of sexual intercourse without contraception ${ }^{[1]}$. Infertility has become a major health problem worldwide, affecting an estimated $8-12 \%$ of couples of reproductive age ${ }^{[2]}$. Among these, male factors may be the primary cause of infertility in approximately $50 \%$ of couples. In addition, infertility can cause serious psychological stress and social problems, which brings considerable economic burden to patients and the health care system ${ }^{[3,4]}$. Thus, it is necessary to facilitate early diagnoses and propose appropriate treatment strategies.

The causes of male infertility can be divided into three categories: congenital, acquired, and idiopathic. In brief, congenital factors such as chromosomal or genetic abnormalities, acquired factors such as varicocele, and idiopathic factors such as psychological stress have been proven to be associated with infertility in men ${ }^{[5]}$. Notably, it is statistically indicated that approximately $10 \%-15 \%$ of male infertility cases can be attributed to genetic mutations ${ }^{[6]}$. Gene variations such as $\mathrm{CFTR}^{[7]}, \mathrm{C} 677 \mathrm{~T}$, and $\mathrm{A} 1298 \mathrm{C}^{[8]}$ might be responsible for male infertility. However, the genetic causes underlying male infertility are complex, with at least 2000 genes participating in spermatogenesis ${ }^{[9]}$. Many of these genes have not been completely identified and characterized, and genetic factors contributing to male infertility remain largely unknown

In recent years, with the development of next-generation sequencing (NGS), significant progress has been achieved in the genetic investigation of humans. NGS can be used to query a large number of genes in a single experiment at a reasonable cost, and targeted NGS methods allow sequencing of genes of interest based on the known disease. Accumulating evidence has demonstrated that NGS is a comprehensive genetic test for male infertility with excellent clinical sensitivity ${ }^{[10,11]}$. In this study, we used targeted NGS to analyze the genetic data of 60 patients diagnosed with abnormal sperm morphology, and eight patients carrying different gene mutations were identified.

\section{Patients And Methods}

\section{Participants and clinical data}

Sixty male patients admitted to the Shandong Provincial Hospital of Traditional Chinese Medicine and Xiyuan Hospital of China Academy of Chinese Medical Sciences between 2018 and 2020 were included in this study. These patients had experienced primary infertility for at least two years. The inclusion criteria included healthy, consensual, idiopathic male infertility, aged 20-45 years with unwanted 1-year childlessness from the same female partner, normal endocrine function, and and with the total number (or concentration) of spermatozoa, and sperm parameters lower than WHO reference limits. Exclusion criteria: testicular abnormalities, cryptorchidism, varicocele, previous genital surgery, or endocrine disorders, gonadal dysfunction test found by physical examination and clinical examination.The Ethics Committee of the Xiyuan Hospital of China Academy of Chinese Medical Sciences approved the study protocol, and all patients provided written informed consent before the examination.

\section{Semen analyses}

Semen analyses were performed to observe sperm motility in the included patients. In brief, semen samples were collected after $72 \mathrm{~h}$ of sexual abstinence. The smears were prepared for each sample and then sent to the clinical laboratory for sperm analysis. Sperm morphology was assessed using the WHO (5.0th edition) guidelines ${ }^{[12]}$

\section{Next generation sequencing (NGS)}

Genomic DNA was extracted from the peripheral blood samples of patients. The Covaris method was used to generate the DNA fragments, and PCR amplification was performed to construct the whole genome library. Next, target gene fragments were captured from the whole genome library via a series of approaches, including specific DNA probe hybridization, which were utilized to construct the target gene library. The library was sequenced on an Illumina 
NextSeq 550Dx instrument, and genetic variation in NGS data was determined using bioinformatics analysis. Specifically, developmental abnormalities, azoospermia, oligospermia, Y chromosome deletion, and CFTR gene-related variants were detected. The pathogenicity of these variants was predicted using the American College of Medical Genetics and Genomics (ACMG) guidelines.

\section{Results}

Of the 60 cases of male infertility, 8 individuals (13\%) were identified as carrying genetic mutations. The clinical characteristics of the patients are summarized in Table 1.

Table 1

The clinical characteristics of eight patients

\begin{tabular}{|c|c|c|c|}
\hline Patient & $\begin{array}{l}\text { Age } \\
\text { (years) }\end{array}$ & Clinical feature & Diagnosis \\
\hline 1 & 30 & NA & Globozoospermia \\
\hline 2 & 29 & NA & Globozoospermia \\
\hline 3 & 31 & NA & Globozoospermia \\
\hline 4 & 32 & $\begin{array}{l}\text { Normal secondary sexual characteristics, testicular volume and vas deferens. } \\
\text { The rate of sperm head abnormalities: } 100 \% \text {. }\end{array}$ & Teratozoospermia \\
\hline 5 & 31 & $\begin{array}{l}\text { Normal secondary sexual characteristics, testicular volume and vas deferens. } \\
\text { Sperm concentration: } 16.0 \text { million } / \mathrm{mL} \text {; the proportion of motile spermatozoa: } 0 \% \text {; the rate of normal } \\
\text { morphology: } 0 \% \text {; and the rate of head deformity: } 100 \% \text {. }\end{array}$ & $\begin{array}{l}\text { Severe asthenospermia } \\
\text { and teratospermia }\end{array}$ \\
\hline 6 & 33 & $\begin{array}{l}\text { Normal secondary sexual characteristics, testicular volume and vas deferens. } \\
\text { Total number of spermatozoa: } 31.24 \text { million; the sperm concentration: } 14.2 \text { million/mL; and the } \\
\text { proportion of motile spermatozoa: } 0.6 \% \text {. }\end{array}$ & Asthenospermia \\
\hline 7 & 30 & Normal secondary sex characteristic, testicular volume, and spermaduct & Oligozoospermia \\
\hline 8 & 39 & NA & Severe asthenospermia \\
\hline
\end{tabular}

Patient 1 was a 30-year-old Chinese man diagnosed with globozoospermia. Genetic testing revealed that the patient carried two heterozygous mutations in SUN5. One variant was a missense mutation, c.1066C > T (p.R356C), located in exon 13 of SUN5 (Fig. 1A), and the other was a frameshift mutation, c.638delC (p.T213fs), located in exon 10 (Fig. 1B). In addition, these two mutations were predicted to be pathogenic by ACMG.

Patient 2 was a 29 year old Chinese man with globozoospermia. NGS analysis showed a 109.73 kb homozygous deletion on chromosome 12 (No. 63952668-64062395) in DPY19L2. Notably, this mutation was also detected in patient 3 (31 years old, globozoospermia).

Patient 4 was a 32 -year-old Chinese man. while the rate of sperm head abnormalities was $100 \%$. Thus, he was diagnosed with teratozoospermia. Two heterozygous mutations of DPY19L2 were detected, including c.1670_1671delinsTT (p.K557I) located in exon 18 (Fig. 1C) and c.255_258del (p.R86Ifs*38) located in exon 1 (Fig. 1D). Of these, c.255_258del was predicted to be pathogenic.

Patient 5 was a 31-year-old man, the semen parameters of this patient were as follows: sperm concentration of $16.0 \mathrm{million} / \mathrm{mL}$, the proportion of motile spermatozoa was $0 \%$, the rate of normal morphology was $0 \%$, and the rate of head deformity was $100 \%$.. He was thus diagnosed with severe asthenospermia and teratozoospermia. Meanwhile, we identified one gene mutation that might be responsible for the teratospermia of this patient; a homozygous variant c. $1159+1 \mathrm{G}>\mathrm{A}$ in intron 7 of $C C D C 4 O$ (Fig. 1E), which was predicted to be pathogenic.

Patient 6 was a 33-year-old Chinese man diagnosed with severe asthenospermia. Semen analysis showed that the total number of spermatozoa was 31.24 million, the sperm concentration was $14.2 \mathrm{million} / \mathrm{mL}$, and the proportion of motile spermatozoa was $0.6 \%$. Next, a likely pathogenic variant in the SEPT12 gene was detected in patient 6. This variant, c.38_43del (p.S13X), is a heterozygous mutation located in exon 2 (Fig. 1F).

Patient 7 was a 30 year old mandiagnosed with asthenospermia. NGS analysis indicated that there were two heterozygous mutations in $D N A H 1$, namely, c.10060_10061insATCT (p.E3354Dfs²8) and c.12278T > C (p.L4093P), which were located in exon 64 (Fig. 1G) and exon 76 (Fig. 1H). Of these, c.10060_10061insATCT was predicted to be a likely pathogenic mutation.

Patient 8 was a 39-year-old man who was diagnosed with severe asthenospermia. Two heterozygous CFAP43 mutations were identified in this patient. One variant was the nonsense mutation c.3658C > T (p.Q1220X) located in exon 28 (Fig. 1I), and the other was a frameshift mutation c.823del (p.H275lfs*3), located in exon 6 (Fig. $1 \mathrm{~J})$. In addition, these two mutations were predicted to be pathogenic.

Overall, 11 different mutations were detected in eight individuals, and detailed information on these genes is presented in Table 2. 
Table 2

Overview of the gene mutations observed in the eight patients

\begin{tabular}{|c|c|c|c|c|c|c|c|c|}
\hline Patient & $\begin{array}{l}\text { Mutated } \\
\text { gene }\end{array}$ & $\begin{array}{l}\text { Genomic position } \\
\text { (bp) }\end{array}$ & cDNA change & Protein change & Transcript & Location & Genotype & pathogenicity \\
\hline \multirow[t]{2}{*}{1} & SUN5 & Chr20:31571674 & c. $1066 \mathrm{C}>\mathrm{T}$ & p.R356C & NM_080675 & Exon 13 & Heterozygous & LP \\
\hline & SUN5 & Chr20:31575557 & c.638delC & p.T213fs & NM_080675 & Exon 10 & Heterozygous & LP \\
\hline 2 & DPY19L2 & $\begin{array}{l}\text { Chr12:63952668- } \\
64062395 \\
\text { deletion }\end{array}$ & NA & NA & NA & NA & Homozygous & NA \\
\hline 3 & DPY19L2 & $\begin{array}{l}\text { Chr12:63952668- } \\
\text { 64062395 } \\
\text { deletion }\end{array}$ & NA & NA & NA & NA & Homozygous & NA \\
\hline \multirow[t]{2}{*}{4} & DPY19L2 & $\begin{array}{l}\text { Chr12:63976240- } \\
63976241\end{array}$ & c.1670_1671delinsTT & p.K557I & NM_173812 & Exon 18 & Heterozygous & VUS \\
\hline & DPY19L2 & $\begin{array}{l}\text { Chr12:64061916- } \\
64061919\end{array}$ & c.255_258del & p.R86lfs*38 & NM_173812 & Exon 1 & Heterozygous & Pathogenic \\
\hline 5 & CCDC40 & Chr17:78024083 & c. $1159+1 \mathrm{GA}$ & NA & NM_017950 & Intron 7 & Homozygous & LP \\
\hline 6 & SEPT12 & $\begin{array}{l}\text { Chr16:4837604- } \\
4837609\end{array}$ & c.38_43del & p.S13X & NM_001154458 & Exon 2 & Heterozygous & LP \\
\hline \multirow[t]{2}{*}{7} & DNAH1 & Chr3:52426487 & c.10060_10061insATCT & p.E3354Dfs*28 & NM_015512 & Exon 64 & Heterozygous & LP \\
\hline & DNAH1 & Chr3:52433054 & c. $12278 \mathrm{~T}>\mathrm{C}$ & p.L4093P & NM_015512 & Exon 76 & Heterozygous & VUS \\
\hline \multirow[t]{2}{*}{8} & CFAP43 & Chr10:105912367 & c. $3658 \mathrm{C}>\mathrm{T}$ & p.Q1220X & NM_025145 & Exon 28 & Heterozygous & LP \\
\hline & CFAP43 & Chr10:105967495 & c.823del & p.H275lfs*3 & NM_025145 & Exon 6 & Heterozygous & LP \\
\hline
\end{tabular}

\section{Discussion}

Male infertility is a multifactorial disease with a significant genetic basis, and sperm abnormalities are one of the causes of infertility in males. In this study, we identified eight patients with abnormal sperm morphology carrying genetic mutations. There were 11 mutations in six genes (SUN5, DPY19L2, CCDC40, SEPT12, DNAH1, and CFAP43) caught our attention that might be involved in male infertility.

NGS analysis showed that the genes SUN5 and DPY19L2 were associated with patients with globozoospermia. Sperm is a polarized cell formed by the head and flagella; thus, head integrity is essential for normal sperm function. Globozoospermia is a severe and rare form of teratozoospermia that is characterized by a spherical sperm head without an acrosome ${ }^{[13]}$. The protein encoded by SUN5 plays a crucial role in the meiotic phase of sperm formation and is specifically expressed in the development of male germ cells. In addition, SUN5 is specifically located at the junction of the head and tail of the sperm ${ }^{[14]}$. A previous study revealed that SUN5 mutations were observed in $37 \%$ of acephalic spermatozoa cases ${ }^{[15]}$, and Elkhatib et al. ${ }^{[16]}$ identified three homozygous deletions of SUN5 in patients with decapitated spermatozoa. However, no direct evidence has indicated a relationship between SUN5 mutations and globozoospermia. Thus, the role of SUN5 in the pathogenesis of globozoospermia requires further investigation. In addition, DPY19L2 is widely reported to be associated with Globozoospermia ${ }^{[17]}$. A homozygous deletion of $200 \mathrm{~kb}$ on chromosome 12 of DPY19L2 might result in Globozoospermia. Meanwhile, evidence suggests that chromosome 12 contains the DPY19L2 locus, and large-scale homozygous deletion might cause the loss of DPY19L2, leading to rounded spermatozoa without acrosomes ${ }^{[19,20]}$. In this study, we found a $109.73 \mathrm{~kb}$ homozygous deletion on chromosome 12 in $D P Y 19 L 2$ of patients with Globozoospermia, which was consistent with previous studies. In addition, we also observed two heterozygous mutations in DPY19L2 that were associated with teratozoospermia, and semen analysis showed that the rate of sperm head abnormalities in this patient was $100 \%$. However, these two variations have not yet been reported. Thus, we speculated that two novel detected intronic mutations, including c.1670_1671delinsTT located in exon 18 and c.255_258del located in exon 1, might be responsible for the abnormal sperm head.

Another gene, $C C D C 40$, was found to be related to severe asthenospermia and teratozoospermia. The highly conserved proteins encoded by $C C D C 40$ are essential for motility cilia, and more than 25 pathogenic mutations in $C C D C 40$ have been identified to participate in primary ciliary dyskinesia (PCD) ${ }^{[21]}$. PCD is characterized by abnormalities in the cilia structure or functional movement. In this patient, semen parameters showed that the proportion of motile spermatozoa was $0 \%$, which may be attributed to the homozygous variant c. $1159+1 \mathrm{G}>\mathrm{A}$ in intron 7 of CCDC40.

Furthermore, we observed that genes such as SEPT12 and CFAP43 were associated with severe asthenospermia. SEPT12 encodes the guanine-nucleotide binding protein, which is related to spermatogenic failure 10 and non-syndromic male infertility due to sperm motility disorder ${ }^{\text {[22] }}$. A published study indicated that the expression level of SEPT12 was lower in testicular tissue of a man with low spermatogenesis than in normal individuals, and downregulation of the SEPT12 gene was associated with an increased number of sperm with abnormal head, neck, and tail morphology, suggesting that SEPT12 is critical in spermatogenesis ${ }^{[23]}$. Kuo et al. reported two missense mutations in SEPT12 related to male infertility presenting as a defective sperm annulus ${ }^{[24]}$. In this study, we also identified a novel heterozygous mutation (c.38_43del) in SEPT12 that might cause severe asthenospermia. Proteins encoded by CFAP43 belong to the cilia- and flagella-related protein family, and can mediate intra-manchette transport and play an important role in sperm flagella formation and 
sperm head shaping ${ }^{[25]}$. Yu et al. ${ }^{[25]}$ also observed that CFAP43 mutations lead to sperm abnormalities and severe asthenospermia. Similarly, we also detected two novel heterozygous mutations in CFAP43 (c.3658C > T and c.823del) associated with severe asthenospermia.

In the present study, two DNAH1 mutations were identified in patients with asthenospermia. DNAH1 encodes an inner dynein arm heavy chain that provides structural support between the radial spokes of the sperm tail and the outer double layer. Mutations in this gene have been related to PCD and various morphological abnormalities of the flagella, leading to male infertility and asthenospermia ${ }^{[26]}$. Sha et al. ${ }^{[27]}$ observed 17 mutations in $D N A H 1$ that contributed to dysplasia of the sperm fibrous sheath and infertility in the Chinese population. In addition, two novel mutations, c.10060_10061insATCT in exon 64 and c. $12278 \mathrm{~T}>\mathrm{C}$ in exon 76, related to asthenospermia, were found in this NGS analysis.

Although these genes have been confirmed to be involved in male infertility, we have discovered several novel genetic mutations. These variations could provide guidance to improve the genetic diagnosis of hereditary infertility. This study had some limitations. The specific mechanisms of the identified genes in male infertility have not yet been investigated. In addition, some mutations in the genes were detected in only one individual. Therefore, a larger study population and longer follow-up of clinical outcomes are needed to verify whether these mutations can be applied in clinical diagnosis.

\section{Conclusion}

Taken together, we identified 11 mutations in six genes (SUN5, DPY19L2, CCDC40, SEPT12, DNAH1, and CFAP43) in male infertility patients. These gene variations are associated with a variety of abnormal sperm morphologies, including globozoospermia, asthenospermia, and teratozoospermia. These findings broaden the genetic causes and pathogenic mechanisms of sperm morphology abnormalities and provide potential diagnostic markers for male infertility patients.

\section{Declarations}

\section{Ethics approval and consent to participate}

Ethical approval was provided by the Shandong Provincial Hospital of Traditional Chinese Medicine in advance of implementation.

\section{Consent for publication}

Written informed consent was obtained from the guardians.

\section{Availability of data and material}

The datasets used during the present study are available from the corresponding author upon reasonable request.

\section{Competing interests}

All authors have no competing interests to declare.

\section{Funding}

All authors have no funding to disclose.

\section{Authors' contributions}

Conceptualization: Zhaowang Gao Investigation: Fengrui Liu Data retrieve: Yong Wang and Zhuozhuo Shi Supervision: Chongfu Zhong and Shengjing Liu Writing original draft: Qinghong Li.

All authors read and approved the final manuscript.

\section{Acknowledgements}

All figures submitted have been created by the authors who confirm that the images are original with no duplication and have not been previously published in whole or in part.

\section{References}

1. ZEGERS-HOCHSCHILD F, ADAMSON G D, DYER S, et al. The International Glossary on Infertility and Fertility Care, 2017 [J]. Hum Reprod. 2017;32(9):1786-801.

2. VANDER BORGHT M. WYNS C. Fertility and infertility: Definition and epidemiology [J]. Clin Biochem. 2018;62:2-10.

3. SCHISTERMAN E F, SJAARDA L A, CLEMONS T, et al. Effect of Folic Acid and Zinc Supplementation in Men on Semen Quality and Live Birth Among Couples Undergoing Infertility Treatment: A Randomized Clinical Trial [J]. Jama. 2020;323(1):35-48.

4. ZHANG T, WANG X, WANG Z, et al. A Diagnostic Model to Improve the Predictability of Natural Pregnancy Potential in Patients with Oligoasthenospermia [J]. Med Sci Monit, 2020, 26(e922316.

5. AGARWAL A, BASKARAN S, PAREKH N, et al. Male infertility [J]. Lancet. 2021;397(10271):319-33. 
6. KRAUSZ C, RIERA-ESCAMILLA A. Genetics of male infertility [J]. Nature reviews Urology. 2018;15(6):369-84.

7. BIENIEK JM, LAPIN C D, JARVI K A. Genetics of CFTR and male infertility [J]. Translational andrology urology. 2021;10(3):1391-400.

8. HE X F HANLJ, YE X H. Methylenetetrahydrofolate reductase C677T and A1298C polymorphisms and male infertility risk: An updated meta-analysis [J]. Med (Baltim). 2020;99(51):e23662.

9. MITCHELL MJ, METZLER-GUILLEMAIN C, TOURE A, et al. Single gene defects leading to sperm quantitative anomalies [J]. Clin Genet. 2017;91(2):20816.

10. ROBAY A, ABBASI S, AKIL A, et al. A systematic review on the genetics of male infertility in the era of next-generation sequencing [J]. Arab journal of urology. 2018;16(1):53-64.

11. CANNARELLA R, PRECONE V, GUERRI G, et al. Clinical Evaluation of a Custom Gene Panel as a Tool for Precision Male Infertility Diagnosis by NextGeneration Sequencing [J]. Life (Basel, Switzerland), 2020, 10(10): 242.

12. MARZANO G, CHIRIACò MS, PRIMICERI E, et al. Sperm selection in assisted reproduction: A review of established methods and cutting-edge possibilities [J]. Biotechnol Adv, 2020, 40(107498.

13. FESAHAT F, HENKEL R. AGARWAL A. Globozoospermia syndrome: An update [J]. Andrologia. 2020;52(2):e13459.

14. BEUROIS J, CAZIN C, KHERRAF Z E, et al. Genetics of teratozoospermia: Back to the head [J]. Best practice \& research Clinical endocrinology \& metabolism, 2020, 34(6): p. 101473.

15. ZHU F, WANG F, YANG X, et al. Biallelic SUN5 Mutations Cause Autosomal-Recessive Acephalic Spermatozoa Syndrome [J]. Am J Hum Genet. 2016;99(4):942-9.

16. ELKHATIB R A, PACI M, LONGEPIED G, et al. Homozygous deletion of SUN5 in three men with decapitated spermatozoa [J]. Human molecular genetics. 2017;26(16):3167-71

17. TAVALAEE M, NOMIKOS M, LAI F A, et al. Expression of sperm PLC and clinical outcomes of ICSI-AOA in men affected by globozoospermia due to DPY19L2 deletion [J]. Reprod Biomed Online. 2018;36(3):348-55.

18. CELSE T, CAZIN C, MIETTON F, et al. Genetic analyses of a large cohort of infertile patients with globozoospermia, DPY19L2 still the main actor, GGN confirmed as a guest player [J]. Hum Genet. 2021;140(1):43-57.

19. ALIMOHAMMADI F, EBRAHIMI NASAB M, RAFAEE A, et al. Deletion of dpy-19 like 2 (DPY19L2) gene is associated with total but not partial globozoospermia [J]. Reproduction, fertility, and development, 2020, 32(8): 727-37.

20. QIU Y, YANG H, LI C, et al. Progress in Research on Sperm DNA Fragmentation [J]. Med Sci Monit, 2020, 26 (e918746.

21. SUI W, HOU X. CHE W, et al. CCDC40 mutation as a cause of primary ciliary dyskinesia: a case report and review of literature [J]. Clin Respir J. 2016;10(5):614-21.

22. SHEN Y R, WANG H Y, KUO Y C, et al. SEPT12 phosphorylation results in loss of the septin ring/sperm annulus, defective sperm motility and poor male fertility [J]. PLoS Genet. 2017;13(3):e1006631.

23. RAFAEE A, MOHSENI MEYBODI A, YAGHMAEI P, et al. Single-nucleotide polymorphism c.474G > A in the SEPT12 gene is a predisposing factor in male infertility [J]. Mol Reprod Dev. 2020;87(2):251-9.

24. KUO Y C, LIN Y H, CHEN H I, et al. SEPT12 mutations cause male infertility with defective sperm annulus [J]. Hum Mutat. 2012;33(4):710-9.

25. YU Y, WANG J. ZHOU L, et al. CFAP43-mediated intra-manchette transport is required for sperm head shaping and flagella formation [J]. Zygote (Cambridge England). 2021;29(1):75-81.

26. YANG X, ZHU D, ZHANG H, et al. Associations between DNAH1 gene polymorphisms and male infertility: A retrospective study [J]. Med (Baltim). 2018;97(49):e13493.

27. LV M, LIU W. CHI W, et al. Homozygous mutations in DZIP1 can induce asthenoteratospermia with severe MMAF [J]. J Med Genet. 2020;57(7):445-53.

\section{Figures}




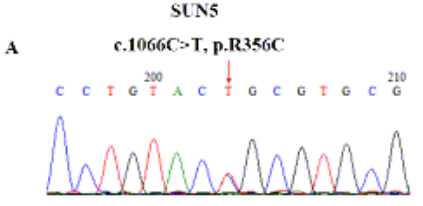

DPY191.2

C.

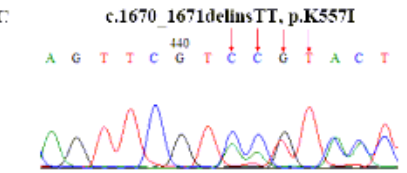

E

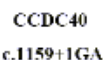

A A A A A G

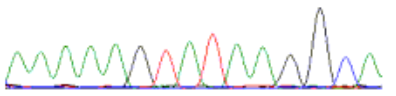

DVAHI

G c.10060_10061insATCT, p.E3354Dfs`28 F

C G C C T A G ${ }^{160} T$ G A C C O

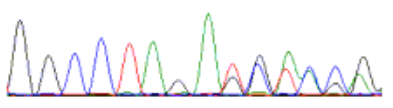

CFAP43

1 c. $3658 \mathrm{C} \approx \mathrm{T}, \mathrm{p} . \mathrm{Q} 1220 \mathrm{X}$
B c.638delC, p.T213fs

G A C I G ${ }_{A}^{140}$ C $\perp$ T T G G G C

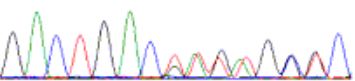

DPY19L2

D c.255_258del, p.R86Ifs $\$ 38$
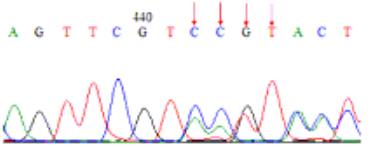

SE:PT12

F c.38 43del, p.\$13X

- AO G ${ }^{110} C T^{\prime} O C O A O O A^{\prime}$

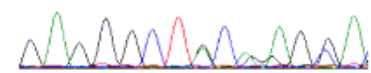

DNAHI

c.12278T $>$ C, p.L4093P

$A T G G$ A C C है G C T G C

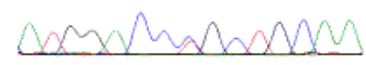

CFAP43

J

c. 823del, p.H275Ifs 3

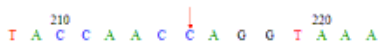

$A \stackrel{130}{A}$ A A G A I $\frac{1}{\mathrm{G}}$ A C C $\mathrm{C}^{130} \mathrm{C}$ C

monallus

Figure 1

The analysis of gene mutation site of the eight individuals identified as carrying genetic mutations. 